Schmerz 2018 $\cdot 32: 356-363$

https://doi.org/10.1007/s00482-018-0302-x

Online publiziert: 5 . Juni 2018

(c) Der/die Autor(en) 2018

CrossMark
P. Kutschar · K. Lex · J. Osterbrink · S. Lorenzl

Institut für Pflegewissenschaft und -praxis, Paracelsus Medizinische Privatuniversität, Salzburg, Österreich

\title{
Morbus Parkinson, Morbus Alzheimer und onkologische Erkrankungen in der stationären Altenhilfe
}

\section{Schmerzhäufigkeit und ausgewählte Versorgungsmerkmale im Vergleich}

\section{Hintergrund und Fragestellung}

In Deutschland leben $27 \%$ aller im Sinne des Pflegeversicherungsgesetzes anerkannten pflegebedürftigen Menschen $(n \approx 780.000)$ in einem der $13.600 \mathrm{Al}$ ten- und Pflegeheime [1]. Sowohl Bewohner als auch die in den Einrichtungen beschäftigten Pflegenden stehen vor vielfältigen Herausforderungen. Häufig leiden Bewohner unter zahlreichen Komorbiditäten wie z. B. Mangelernährung, Schmerzen, Atemnot, Depression, physischer Gebrechlichkeit und - im vorliegenden Kontext von besonderer Relevanz - demenziellen Erkrankungen [2-4]. Die verbleibende Lebens- bzw. „Sterbezeit“ ist oft relativ kurz, weshalb stationäre Altenpflegeeinrichtungen auch als „Orte höchster Sterbeintensität" bezeichnet werden [5].

Einen nicht unbeträchtlichen Anteil an typischen Altenheimpopulationen stellen Menschen mit onkologischen Erkrankungen dar. Internationale Studien schätzen Prävalenzen zwischen 9 und $15 \%$ der Altenheimbewohner (AHB) [6]. In der Bevölkerung liegt das Erkrankungsrisiko bei Männern über 65 Jahre in den darauffolgenden 10 Jahren bei 1:3; bei Frauen bei 1:5 [7]. Eine Hauptbelastung für Tumorpatienten sind Schmerzen: Bei bis $\mathrm{zu} 80 \%$ treten sie im fortgeschrittenen Krankheitsstadium auf [8]. Drei Viertel aller Krebstodesfälle treten bei Menschen auf, die älter als 65 Jahre alt sind [9]. Bei dieser Bewohnergruppe gestalten sich Entscheidungsfindungsprozesse bezüglich des eigenen Lebensendes häufig als herausfordernd und komplex: Es ist durchaus möglich, dass sich Präferenzen im Krankheitsverlauf verändern oder dass sich kurzfristig der Allgemeinzustand verbessert, etwa weil nebenwirkungsreiche oder nicht (mehr) indizierte Pharmakotherapien abgesetzt werden [10].

Es ist weiter davon auszugehen, dass ca. zwei Drittel aller deutschen $\mathrm{AHB}$ an einer leichten, mittelschweren oder schweren Form einer demenziellen Erkrankung leiden [11]. Schätzungen zufolge leben in Deutschland etwa 1,5 Mio. Menschen mit einer Demenzerkrankung. Bis 2030 könnte die Zahl an einer Demenz erkrankter Personen auf rund 3 Mio. Menschen anwachsen [12]. Regelhafte Symptome und pflegerischen Bedarf begründende Probleme bei fortschreitender Demenz bestehen in Verwirrtheit, Depression, Obstipation, Schluckstörungen, Schwierigkeiten im gegenseitigen Verstehen, Unterernährung und besonders Schmerz von Betroffenen [3, 9, 13-15]. Hier haben vor allem ältere Menschen mit fortgeschrittenen kognitiven Beeinträchtigungen ein stark erhöhtes Risiko für eine ungenügende Schmerztherapie. Aufgrund eingeschränkter kommunikativer Fähigkeiten ist es Betroffenen häufig nicht möglich, Schmerzen adäquat zu kommu- nizieren. Deshalb gelingt es Pflegenden und Ärzten oft nicht, angemessen auf etwaige Schmerzzustände $\mathrm{zu}$ reagieren [9]. Die Schätzungen zur Überlebenszeit von an Demenz erkrankten Bewohnern variieren. Als mittlere Überlebensdauer (Median) von der Diagnose bis zum Eintreten des Todes wird von ca. 8 Jahren ausgegangen [9]. Für Deutschland wird die mittlere Überlebenszeit nach „Manifestation der Erkrankung " mit 3,3 Jahren geschätzt [13]. Gerade vor dem Hintergrund demenzieller Erkrankungen zeigt sich der deutliche Bedarf an PalliativeCare-Konzepten in Altenheimen: Demenz ist eine unheilbare, chronisch fortschreitende, letztlich zum Tod führende Erkrankung [13] und insofern haben Betroffene „bereits vom ersten Augenblick an Anspruch auf Palliative Care“ [14].

Morbus Parkinson gilt mit einer Prävalenz von $110-180$ in 100.000 bevölkerungsbezogen als die zweithäufigste neurodegenerative Erkrankung [16]. In Alten- und Pflegeheimen hingegen lebt einer von 10 Bewohnern mit der Diagnose Morbus Parkinson. Nach der Diagnosestellung beträgt die mittlere Lebenserwartung etwa 15 Jahre [17]. Aus der Literatur ist hinreichend bekannt, dass Menschen, bei denen Parkinson diagnostiziert worden ist, enorme unerfüllte Palliative-Care-Bedürfnisse haben. Die Symptombelastung ist ebenso groß wie bei Tumorpatienten, wobei Pallia- 
tive-Care-Bedarfe von Menschen mit Morbus Parkinson aber viel seltener als bei „klassischen onkologischen Patienten" durch lokale Strukturen abgedeckt werden [18]. Was die pflegerische und medizinische Versorgung von an Morbus Parkinson erkrankten Bewohnern zusätzlich erschwert, ist eine „Parkinson-assoziierte Multimorbidität", welche sich aber zum Lebensende kaum vermeiden lässt und zudem meistens als schmerzinduzierend anzunehmen ist [10].

Bei oben angeführten Erkrankungsbildern scheint folglich die Orientierung an explizit inklusiven Palliative-CareKonzepten auch im Altenheimkontext unumgänglich. Die zentralen Ansprüche der European Association for Palliative Care (EAPC), die Palliative Care als „aktive, umfassende Versorgung und Pflege von Patienten, deren Erkrankung nicht auf eine kurative Behandlung anspricht“ definiert und dabei „Kontrolle von Schmerzen oder anderen Symptomen ..." hervorhebt, sind dabei von höchster Relevanz [19]. Weitere Bedeutung erhält der interdisziplinäre Ansatz, indem er sich deutlich auf Betroffene, deren Familien und die Gemeinschaft bezieht und sich als unabhängig vom spezifischen Versorgungssetting positioniert. Palliative Care hat dabei das erklärte Ziel, „die beste Lebensqualität bis zum Tod zu gewährleisten“" [19]. Bewährt hat sich eine derartige „early integration" von Palliative Care bei der Betreuung onkologisch erkrankter Menschen [20]. Auch in der Begleitung von Menschen mit Morbus Parkinson oder demenziellen Erkrankungen empfehlen Experten ein frühzeitiges Einbeziehen palliativer Therapieoptionen [16]. Insgesamt gilt es inzwischen als weitgehend unumstritten, dass die letzte Lebensphase von Menschen mit Morbus Parkinson, Alzheimer-Demenz und Tumorerkrankung zunehmend in Altenheimen erlebt wird und daher der ,end-of-life care “ für diese Menschen in den Heimen wissenschaftlich wie praxisbezogen vermehrte Aufmerksamkeit zukommen muss [6, 21, 22].

\section{Fragestellung}

Ziel dieser explorativen Sekundärdatenanalyse ist es, ausgewählte Unterschiede in Bezug auf biometrische und pflegerische Charakteristika und der Schmerzsituation zwischen Altenheimbewohnern (AHB) mit Morbus Parkinson, Alzheimer-Demenz und onkologischen Erkrankungen darzustellen und vor dem Hintergrund von pflegerischen und palliativen Versorgungsaspekten zu diskutieren. Anhand von empirischen Daten aus der stationären Altenhilfe wurde untersucht, ob sich AHB mit Parkinson, AHB mit Alzheimer und AHB mit Tumor in den Merkmalen Geschlecht, Lebensalter, kognitive Leistungsfähigkeit, Komorbiditäten, Pflegestufe, Pflegesetting vor Einzug ins Altenheim, Aufenthaltsdauer in Jahren und Schmerzsituation (Selbst- und/ oder Fremdeinschätzung) voneinander unterscheiden.

\section{Studiendesign und Untersuchungsmethoden}

\section{Design}

Die ursprünglichen Daten entstammen dem Versorgungsforschungsprojekt „Aktionsbündnis Schmerzfreie Stadt Münster“ (2010-2014). In dieser Studie wurde das interdisziplinäre Schmerzmanagement in verschiedenen Versorgungseinrichtungen (Krankenhäuser, Schmerzpraxen, ambulante Pflegedienste, Hospize, Altenheime) einer ganzen Stadt untersucht. Einen Teilbereich stellte die Untersuchung des Schmerzmanagements bei chronischen und akuten Schmerzen in 13 Altenheimen der nordrhein-westfälischen Stadt Münster im Rahmen einer Ex-post-facto-Studie mit Prä-post-Test dar. Der Istzustand des umgesetzten Schmerzmanagements wurde im Prätest mittels Strukturdatenanalysen, Online-Surveys der in den Einrichtungen tätigen Pflegekräfte und Befragungen wie Beobachtungen von Bewohnern der eingeschlossenen Altenheime erhoben. Auf Basis dieser Ergebnisse erfolgten dann einrichtungsspezifische Interventionen. Diese Interventionen sind nicht Gegenstand der vorliegenden Arbeit. De- taillierte Informationen $\mathrm{zu}$ den Zielen und Methoden der Hauptstudie wurden mehrfach veröffentlicht (z. B. [4]).

\section{Datenerhebung}

Von insgesamt 32 Altenheimen der Stadt Münster (Stand: Juni 2010) erklärten sich 13 Einrichtungen zur Teilnahme am Forschungsprojekt bereit. In jeder Einrichtung wurden strukturelle Daten mittels Einrichtungsdatenblatt erhoben. Medizinische und biometrische Charakteristika wurden aus den bewohnerspezifischen Pflegedokumentationen entnommen. AHB wurden mit standardisierten Face-to-face-Interviews durch Studienassistenten befragt bzw. mittels spezifischer Fremdeinschätzungsinstrumente beobachtet. Die eingesetzten Studienassistenten verfügten über eine pflegerische Grundausbildung bzw. waren Studierende der Pflegewissenschaft oder Gesundheitswissenschaften. Vor der Datenerhebung nahmen die Studienassistenten an einer ganztägigen Interviewerschulung teil. Der Prätest fand von September 2010 bis April 2011, der Posttest von Juli 2012 bis April 2013 statt. Pro Einrichtung dauerte die Datenerhebung zwischen 4 und 6 Wochen.

\section{Instrumente}

Soziodemografische und medizinisch wie pflegerisch relevante Merkmale wurden aus den bewohnerspezifischen Pflegedokumentationen übertragen. Der Status der kognitiven Leistungsfähigkeit wurde mittels MMST erfasst und zur Stratifikation in 3 Subgruppen kognitiver Leistungsfähigkeit genutzt [23]. Die Erfassung der subjektiven Schmerzsituation orientierte sich sodann an der bewohnerindividuellen kognitiven Leistungsfähigkeit. Bewohner mit keiner oder leichter kognitiver Beeinträchtigung (MMST 19-30 Punkte) wurden per validiertem Selbstauskunftsinstrument der 5-stufigen „verbalen Rangskala“ (VRS-5; [24]) befragt. Bei Bewohnern mit stark fortgeschrittener kognitiver Beeinträchtigung (MMST 0-9 Punkte) wurde das Fremdeinschätzungsinstrument BESD (Beurteilung von Schmerzen bei Demenz; [25]) eingesetzt. Höhere 
Schmerz 2018 -32:356-363 https://doi.org/10.1007/s00482-018-0302-x

(c) Der/die Autor(en) 2018

P. Kutschar · K. Lex · J. Osterbrink · S. Lorenzl

Morbus Parkinson, Morbus Alzheimer und onkologische Erkrankungen in der stationären Altenhilfe. Schmerzhäufigkeit und ausgewählte Versorgungsmerkmale im Vergleich

\section{Zusammenfassung}

Hintergrund. Vorhandene Schmerzmanagement- und Palliativversorgungsstrukturen sind in der stationären Altenhilfe von großer Relevanz. Während eine professionelle Schmerzbehandlung und palliativversorgerische Maßnahmen bei Bewohnern mit onkologischen Erkrankungen häufig stattfinden, ist dies bei Bewohnern mit neurologischen Erkrankungen oft nicht der Fall. Diese potenzielle Unterversorgung ist dann besonders herausfordernd, wenn die Interaktion mit betroffenen Bewohnern durch Einbußen der kognitiven Leistungsfähigkeit weiter erschwert wird.

Ziel der Arbeit. Vergleich ausgewählter Versorgungsmerkmale zwischen Bewohnern mit Morbus Parkinson, Morbus Alzheimer und Tumorerkrankung.
Methodik. Sekundärdatenanalyse von Bewohnerbefragungs- und Pflegedokumentationsdaten aus 13 Altenheimen der Studie "Aktionsbündnis Schmerzfreie Stadt Münster". Ergebnisse. Im Vergleich zeigen sich bei Bewohnern mit Alzheimer signifikant stärker ausgeprägte kognitive Beeinträchtigungen, weniger zusätzliche schmerzassoziierte Diagnosen, kürzere Verweildauern im Altenheim und mehr Schmerzhinweise als bei Bewohnern mit Parkinson und Tumorerkrankungen.

Diskussion. Das generell als eher hoch zu bezeichnende Schmerzauftreten verdeutlicht die prinzipielle Notwendigkeit eines angemessenen Schmerzassessments und einer interprofessionellen schmerztherapeutischen Betreuung. Bei Bewohnern mit Parkinson und
Alzheimer ist das umso wichtiger, scheinen bei dieser wachsenden Bewohnergruppe doch spezifische Implementierungen und Maßnahmen nötig zu sein. Der Konzeption, Umsetzung und wissenschaftlichen Evaluation von spezifisch adaptierten Strategien und Maßnahmen, die auf die Bedürfnisse der Bewohner abgestimmt sind, sollte zukünftig verstärkte (pflege)praktische wie (pflege)wissenschaftliche Aufmerksamkeit zukommen.

Schlüsselwörter

Schmerzmanagement im Altenheim . Kognitive Leistungsfähigkeit · Morbus Parkinson · Morbus Alzheimer · Tumor

\section{Parkinson's disease, Alzheimer's disease and oncological diseases in residential geriatric care. Pain frequency and selected healthcare features in comparison}

\section{Abstract}

Background. Adequate pain management and palliative care structures are of significant importance in residential nursing homes. Whilst professional pain treatment and palliative care measures are frequently implemented for residents with oncological diseases, this is often not the case for residents with neurological disorders. Such a potential undertreatment is even more challenging when the means of interaction and communication with affected persons are aggravated by impairments in cognitive function.

Objective. To examine differences in selected health care service characteristics between nursing home residents with Parkinson's disease, Alzheimer's disease and residents diagnosed with cancer.

Material and methods. Secondary data analysis of residents' survey and medical record data from 13 nursing homes as part of the study "Action Alliance Pain-free City Münster".

Results. Compared with residents with Parkinson's disease and cancer, nursing home residents with Alzheimer's disease exhibited significantly more severe impairment in cognitive function, less additional painassociated diagnoses, shorter length of stay in nursing homes and more indications of pain. Conclusion. The generally high level of pain in all observed residents elucidates the principle necessity of adequate pain assessment and an interprofessional pain treatment. Furthermore, there seems to be a still unmet need for specifically adapted pain management strategies especially for the steadily increasing number of people with Parkinson's disease and Alzheimer's disease living in nursing homes. This should be a future high priority task for (nursing) practice and research against the background of the vulnerability of nursing home populations.

Keywords

Pain management in nursing homes .

Cognitive function · Parkinson's disease .

Alzheimer's disease · Cancer
BESD-Punktwerte $\left(\mathrm{BESD}_{\min -\max }=0-10\right)$ bedeuten dabei mehr Hinweise auf das Vorhandensein von Schmerzen. Bewohner mit moderater kognitiver Beeinträchtigung (MMST 10-18 Punkte) wurden standardisiert befragt (VRS) sowie per Fremdeinschätzung beobachtet (BESD). Um die Schmerzsituation auch über die 3 Subgruppen hinweg vergleichen zu können, wurde aus den Ergebnissen von VRS und/oder BESD die Variable „Schmerzhinweis“ operationalisiert. Als vorliegender Schmerzhinweis galt, wenn bei Bewohnern mit keiner oder leichter kognitiver Beeinträchtigung mindestens "leichter Schmerz" (VRS) angegeben wurde. In der Bewohnergruppe mit MMST-Werten zwischen 0 und 9 wurde als Cut-off ein BESD-Score ab „2" herangezogen [26]. Schmerzhinweise bei Bewohnern mit moderaten kognitiven Beeinträchtigungen wurden dann als solche angenommen, wenn entweder leichte Schmerzen laut Selbstauskunft oder ein BESD-Wert über oben genanntem Cut-off erfasst wurde. Der Vergleich zwischen den Bewohnergruppen, bei denen Schmerzen mittels BESD erfasst wurden, wird mit dem Punktwert sowie dem Anteil an Bewohnern über Cut-off durchgeführt.

\section{Studienteilnehmer}

Als Studienpopulation galten alle Bewohner der inkludierten Altenheime. Vor 


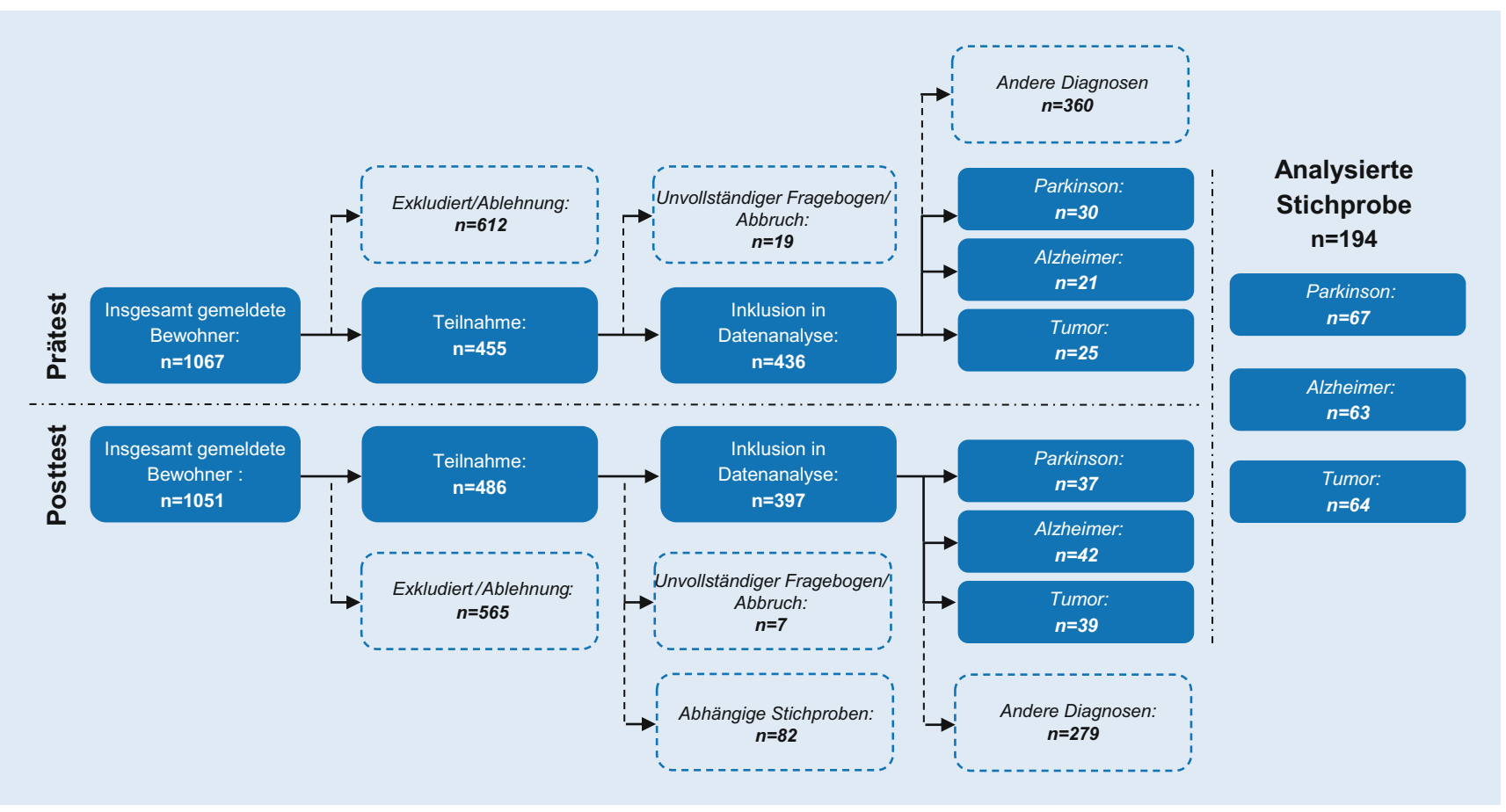

Abb. 1 A Flowchart zur Ausschöpfung

dem Hintergrund der geplanten Vollerhebung war angestrebt, sämtliche $\mathrm{Be}$ wohner einzuschließen, die mindestens 65 Jahre alt waren, dauerhaft in der Einrichtung lebten, ausreichende Kenntnisse der deutschen Sprache hatten und sich in keiner akut lebensbedrohlichen Situation befanden. In der vorliegenden Sekundärdatenanalyse werden nur jene Altenheimbewohner eingeschlossen, die die dokumentierten Diagnosen Morbus Parkinson, Alzheimer-Demenz oder Tumorerkrankung aufwiesen.

\section{Statistische Datenanalyse}

Sämtliche Analysen wurden mit IBM SPSS 22 durchgeführt. Zur Darstellung der Stichprobencharakteristika wurden gängige Maße der deskriptiven Statistik eingesetzt. Zur Analyse von Unterschieden in den Anteilen nominalskalierter Variablen wurden $\chi^{2}$ Anpassungstests mit Fisher-Yates-Korrektur genutzt. Gruppenunterschiede bei Variablen ordinaler Skalierung wurden mittels Kruskal-Wallis-H-Test getestet. Die Prüfung auf statistisch relevante Differenzen bei metrischen Variablen erfolgte anhand einfaktorieller Varianzanalysen (ANOVA) mit Bonferroni-
Korrektur. Das a-Fehler-Niveau wurde a priori mit $p<0,05$ festgesetzt.

\section{Ethikvotum}

Die Studie wurde von der Ethik-Kommission der Medizinischen Fakultät der Westfälischen Wilhelms-Universität genehmigt (Aktenzeichen 2010-010-f-S).

\section{Ergebnisse}

\section{Ausschöpfung}

Im Prätest wurden insgesamt 1067 Bewohner als Auswahlgesamtheit gemeldet. Davon lehnten 612 AHB bzw. deren gesetzliche Betreuer die Teilnahme an der Studie ab oder wurden aufgrund der Ein-/ Ausschlusskriterien exkludiert. Nach der Datenbereinigung lagen 436 gültige Fälle vor. Die Ausschöpfung gestaltete sich im Posttest ähnlich: Von 1067 gemeldeten Bewohnern nahmen 486 an der Studie teil, wobei 397 Bewohner inkludiert wurden. Die vorliegenden Analysen schließen nur AHB mit Parkinson-, Alzheimer- oder Tumordiagnosen ein. Über den Prä- und Posttest hinweg konnten in Summe 194 Altenheimbewohner (20,8\% von in die Datenanalyse einge- schlossenen AHB) mit mindestens einer der drei interessierenden Diagnosen inkludiert werden. Die Verteilung dieser Diagnosen ist relativ ausgeglichen: $34,5 \%(n=67)$ wiesen eine Morbus-Parkinson-Diagnose auf, 32,5\% $(n=63)$ eine Alzheimer-Demenz und 33,0\% $(n=64)$ eine Tumorerkrankung. $\bullet$ Abb. 1 stellt die Ausschöpfung für den Prä- und Posttest dar.

\section{Stichprobendeskription}

- Tab. 1 zeigt soziodemografische und pflegerelevante Stichprobeneigenschaften für Altenheimbewohner mit Parkinson $\left(\mathrm{AHB}_{\text {Parkinson }}\right)$, $\mathrm{AHB}$ mit Alzheimer $\left(\mathrm{AHB}_{\text {Alzheimer }}\right)$ und $\mathrm{AHB}$ mit Tumorerkrankungen ( $\left.\mathrm{AHB}_{\text {Tumor }}\right)$.

In der Bewohnergruppe mit Morbus Parkinson waren drei Viertel aller AHB weiblich. Das mittlere Alter betrug 84,9 $( \pm 6,6)$ Jahre. Im Durchschnitt erreichten die $\mathrm{AHB}_{\text {Parkinson }}$ einen MMST-Wert von $13,2( \pm 10,8)$ Punkten. Bei mehr als $55 \%$ lag eine dokumentierte Pflegestufe von Stufe II oder höher vor. Die häufigste Begleitdiagnose war Demenz $(53,7 \%)$. Durchschnittlich wiesen die Untersuchungspersonen $1,6( \pm 1,1) \mathrm{zu}$ sätzliche Diagnosen auf. Ca. $65 \%$ aller 
Tab. 1 Deskriptive Kennwerte der Stichprobe getrennt nach AHB mit Parkinson/Alzheimer/

Tumor

Deskriptive Statistiken; Mittelwert \pm Standardabweichung, Anteil in \% ( $n$ )

\begin{tabular}{|c|c|c|c|}
\hline & AHB mit Parkinson & AHB mit Alzheimer & AHB mit Tumor \\
\hline Weiblich & $74,6 \%(20)$ & $82,5 \%(52)$ & $81,0 \%(51)$ \\
\hline Alter in Jahren & $84,9 \pm 6,6$ & $84,6 \pm 6,5$ & $85,3 \pm 6,4$ \\
\hline MMST-Punkte & $13,2 \pm 10,8$ & $12,6 \pm 9,9$ & $16,7 \pm 9,9$ \\
\hline \multirow[t]{5}{*}{ Pflegestufe } & Keine $3,0 \%(2)$ & Keine $9,7 \%(6)$ & Keine $11,1 \%(7)$ \\
\hline & Stufe I 40,3 \% (27) & Stufe I 40,3\% (25) & Stufe I 49,2 \% (31) \\
\hline & Stufe II $29,9 \%$ (20) & Stufe II 35,5\% (22) & Stufe II 31,7\% (20) \\
\hline & Stufe III $26,9 \%$ (18) & Stufe III 11,3\% (7) & Stufe III 7,9\% (5) \\
\hline & Stufe III+ k.A. & Stufe III+ 3,2\% (2) & Stufe III+ k.A. \\
\hline \multirow{3}{*}{$\begin{array}{l}\text { Weitere } \\
\text { Diagnosen }^{\mathrm{a}}\end{array}$} & DEM 53,7\% (36) & DEP $25,4 \%(16)$ & OST $26,6 \%$ (17) \\
\hline & DEP $25,4 \%(17)$ & KHK $12,7 \%$ (8) & DEM 25,0\% (16) \\
\hline & FRA $17,9 \%(12)$ & OST $12,7 \%(8)$ & FRA $23,4 \%(15)$ \\
\hline $\begin{array}{l}\text { Anzahl weiterer } \\
\text { Diagnosen }\end{array}$ & $1,6 \pm 1,1$ & $0,8 \pm 0,8$ & $1,5 \pm 0,9$ \\
\hline \multirow{4}{*}{$\begin{array}{l}\text { Transfer von ... } \\
\text { bei Ersteinzug }\end{array}$} & Zu Hause 65,1 \% (41) & Zu Hause $86,2 \%$ (50) & Zu Hause $86,7 \%$ (52) \\
\hline & Krankenhaus 14,3\% (9) & Krankenhaus 8,6\% (5) & Krankenhaus 5,0\% (3) \\
\hline & Reha $4,8 \%$ (3) & Reha k.A. & Reha 3,3\% (2) \\
\hline & Andere $15,9 \%(10)$ & Andere $5,2 \%(3)$ & Andere $5,0 \%(3)$ \\
\hline $\begin{array}{l}\text { Aufenthaltsdauer } \\
\text { in Jahren }\end{array}$ & $3,7 \pm 4,2$ & $2,1 \pm 2,2$ & $2,9 \pm 2,9$ \\
\hline \multirow{5}{*}{$\begin{array}{l}\text { Ruheschmerz } \\
\text { (VRS) }\end{array}$} & Keiner $52,6 \%(20)$ & Keiner 62,2 \% (23) & Keiner 47,8\% (22) \\
\hline & Leicht 15,8\% (6) & Leicht 16,2\% (6) & Leicht 21,7\% (10) \\
\hline & Mittelstark $21,1 \%$ (8) & Mittelstark $18,9 \%$ (7) & Mittelstark 15,2\% (7) \\
\hline & Stark $10,5 \%(4)$ & Stark $2,7 \%(1)$ & Stark 10,9\% (5) \\
\hline & Unerträglich k.A. & Unerträglich k.A. & Unerträglich 4,3 \% (2) \\
\hline \multirow{5}{*}{$\begin{array}{l}\text { Belastungs- } \\
\text { schmerz (VRS) }\end{array}$} & Keiner $28,9 \%(11)$ & Keiner 47,2 \% (17) & Keiner 30,4 \% (14) \\
\hline & Leicht $21,1 \%$ (8) & Leicht $19,4 \%$ (7) & Leicht 23,9\% (11) \\
\hline & Mittelstark 28,9\% (11) & Mittelstark 19,4\% (7) & Mittelstark 29,9\% (11) \\
\hline & Stark 21,1 \% (8) & Stark $11,1 \%(4)$ & Stark $17,4 \%(8)$ \\
\hline & Unerträglich k.A. & Unerträglich 2,8 \% (1) & Unerträglich 4,3 \% (2) \\
\hline BESD-Punkte & $1,7 \pm 1,9$ & $3,8 \pm 2,6$ & $2,8 \pm 2,5$ \\
\hline $\mathrm{BESD} \geq 2^{\mathrm{b}}$ & $47,1 \%(16)$ & $84,8 \%(28)$ & $57,7 \%(15)$ \\
\hline \multirow[t]{2}{*}{ Schmerzhinweis $^{c}$} & Nein 36,4\% (24) & Nein $24,7 \%$ (16) & Nein 31,3 \% (20) \\
\hline & Ja 63,6\% (42) & Ja 73,3\% (44) & Ja 68,8\% (44) \\
\hline Stichprobengröße & $n=67$ & $n=63$ & $n=64$ \\
\hline \multicolumn{4}{|c|}{ 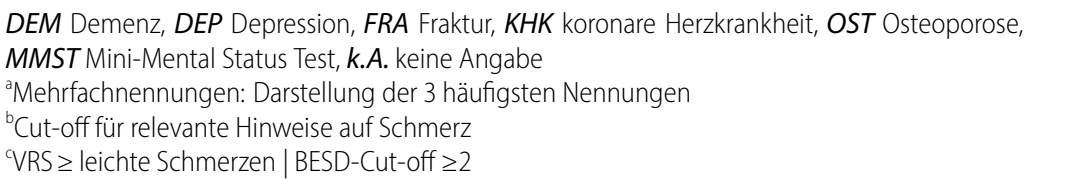 } \\
\hline
\end{tabular}

AHB Parkinson wurden von zu Hause in die Einrichtung transferiert. Die mittlere Aufenthaltsdauer lag bei 3,7 $( \pm 4,2)$ Jahren. Jeder zweite $\mathrm{AHB}_{\text {Parkinson gab }}$ zumindest leichte Ruheschmerzen an, 7 von 10 Befragten gaben an, mindestens leichte Belastungsschmerzen wahrzunehmen. Unter den 34 Bewohnern, die mittels BESD beobachtet wurden, zeig- te sich ein Durchschnittsscore von 1,7 $( \pm 1,9)$ Punkten. Der Anteil von Personen mit vorliegenden Schmerzhinweisen beträgt ca. $64 \%$.

Bei AHB $_{\text {Alzheimer }}$ betrug der Anteil von Bewohnerinnen knapp $83 \%$. Im Mittel waren die Bewohner 84,6 $( \pm 6,5)$ Jahre alt. Der errechnete MMST-Wert lag bei 12,6 $( \pm 9,9)$ Punkten. Eine Pflegestufe von II oder höher kam bei der Hälfte der Bewohner vor. Bei einem Viertel der untersuchten Personen wurde Depression als weitere Diagnose ausgewiesen. Durchschnittlich wurde knapp weniger als eine weitere Diagnose $(0,8 \pm 0,8)$ dokumentiert. $86 \%$ der AHB lebten vor dem Einzug ins Altenheim zu Hause. Der Mittelwert bei der Aufenthaltsdauer betrug 2,1 $( \pm 2,2)$ Jahre. In dieser Gruppe berichteten $38 \%$ der Befragten einen mindestens leichten Ruhebzw. $53 \%$ einen mindestens leichten Belastungsschmerz. Das Instrument BESD wurde bei $33 \mathrm{AHB}_{\text {Alzheimer }}$ eingesetzt. Der BESD-Score betrug 3,8 $( \pm 2,6)$ Punkte. Bei drei Viertel der untersuchten Menschen mit Alzheimer-Diagnose zeigten sich relevante Schmerzhinweise.

Vier von $5 \mathrm{AHB}_{\text {Tumor }}$ waren weiblich. Das Durchschnittsalter betrug 85,3 $( \pm 6,4)$ Jahre. Im Mittel wurden MMSTWerte von 16,7 $( \pm 9,9)$ Punkten erfasst. Eine Pflegestufe von II oder höher hatten ca. $40 \%$ der Bewohner. Mit einem Anteil von $27 \%$ war Osteoporose die am häufigsten genannte Zusatzdiagnose. In dieser Gruppe wurden gesamt betrachtet durchschnittlich $1,5( \pm 0,9)$ weitere Diagnosen dokumentiert. Zu Hause lebten vor dem Transfer ins Altenheim nahezu $87 \%$ aller $\mathrm{AHB}_{\text {Tumor. }}$ Die durchschnittliche Aufenthaltsdauer betrug $2,9( \pm 2,9)$ Jahre. Leichte, mittelstarke, starke oder unerträgliche Ruheschmerzen äußerten ca. $52 \%$. Hinsichtlich der Belastungsschmerzen waren dies fast $70 \%$. Unter jenen $26 \mathrm{AHB}_{\text {Tumor, die ei- }}$ ner Fremdeinschätzung von Schmerzen unterzogen wurden, lag der betreffende Mittelwert bei 2,8 $( \pm 2,5)$ Punkten laut BESD. Als wahrscheinlich von Schmerzen betroffen galten in dieser Gruppe knapp $69 \%$.

\section{Merkmale mit signifikanten Unterschieden zwischen AHBParkinson, $\mathrm{AHB}_{\text {Alzheimer }}$ und AHBTumor}

Die signifikanten Ergebnisse der Gruppenvergleiche sind in $\bullet$ Tab. 2 angeführt. Es finden sich keine signifikanten Unterschiede zwischen den untersuchten Subgruppen hinsichtlich Geschlecht, Alter, Ruhe- und Belastungsschmerz sowie der verallgemeinerten Schmerz- 
Tab. 2 Darstellung signifikanter Unterschiede von Merkmalen zwischen AHB mit Parkinson/ Alzheimer/Tumor

\begin{tabular}{|c|c|c|c|c|}
\hline \multicolumn{5}{|c|}{ Statistische Tests; Mittelwert, mittlerer Rang, \%, Teststatistik, $p(n)$} \\
\hline & $\begin{array}{l}\text { AHB mit Par- } \\
\text { kinson }\end{array}$ & $\begin{array}{l}\text { AHB mit Alzhei- } \\
\text { mer }\end{array}$ & AHB mit Tumor & Teststatistik, $p(n)$ \\
\hline MMST-Punkte ${ }^{b}$ & 13,2 & 12,6 & 16,7 & $\begin{array}{l}\mathrm{F}=2,925, p=0,056^{\mathrm{d}} \\
(194)\end{array}$ \\
\hline Pflegestufe $^{c}$ & 109,0 & 95,7 & 83,9 & $\begin{array}{l}x^{2}=7,502, p=0,023 \\
(192)\end{array}$ \\
\hline $\begin{array}{l}\text { Anzahl } \\
\text { weiterer } \\
\text { Diagnosen }^{\text {b }}\end{array}$ & 1,60 & 0,79 & 1,52 & $\begin{array}{l}\mathrm{F}=13,859, p<0,001 \\
(194)\end{array}$ \\
\hline \multirow{4}{*}{$\begin{array}{l}\text { Transfer } \\
\text { von ... bei } \\
\text { Ersteinzug }^{\text {a }}\end{array}$} & Zu Hause $65,1 \%$ & Zu Hause $86,2 \%$ & Zu Hause $86,7 \%$ & \multirow{4}{*}{$\begin{array}{l}x^{2}=12,361, p=0,036 \\
(181)\end{array}$} \\
\hline & $\begin{array}{l}\text { Krankenhaus } \\
14,3 \%\end{array}$ & $\begin{array}{l}\text { Krankenhaus } \\
8,6 \%\end{array}$ & $\begin{array}{l}\text { Krankenhaus } \\
5,0 \%\end{array}$ & \\
\hline & Reha $4,8 \%$ & Reha k.A. & Reha $3,3 \%$ & \\
\hline & Andere $15,9 \%$ & Andere $5,2 \%$ & Andere $5,0 \%$ & \\
\hline $\begin{array}{l}\text { Aufenthalts- } \\
\text { dauer in } \\
\text { Jahren }^{b}\end{array}$ & 3,65 & 2,14 & 2,89 & $\begin{array}{l}\mathrm{F}=3,526, p=0,031 \\
(193)\end{array}$ \\
\hline $\mathrm{BESD}^{\mathrm{b}}$ & 1,74 & 3,76 & 2,81 & $\begin{array}{l}\mathrm{F}=6,265, p=0,003 \\
(93)\end{array}$ \\
\hline $\mathrm{BESD} \geq 2^{\mathrm{a}}$ & $47,1 \%$ & $84,8 \%$ & $57,7 \%$ & $x^{2}=10,825, p=0,004$ \\
\hline $\begin{array}{l}\text { Stichproben- } \\
\text { größe }\end{array}$ & $n=67$ & $n=63$ & $n=64$ & $n=194$ \\
\hline \multicolumn{5}{|c|}{$\begin{array}{l}\text { MMST Mini-Mental Status Test, BESD Beurteilung von Schmerzen bei Demenz, } k . A \text {. keine Angabe } \\
{ }^{2} X^{2} \text { mit Fisher-Yates-Korrektur } \\
{ }^{{ }^{6}} \text { ANOVA mit Bonferroni } \\
\text { KKruskal-Wallis-H-Test } \\
{ }^{d} \text { Trend }(p<0,10)\end{array}$} \\
\hline
\end{tabular}

hinweise. Es zeigt sich jedoch, dass $\mathrm{AHB}_{\text {Tumor }}$ einen tendenziell höheren MMST-Wert aufweisen als $\mathrm{AHB}_{\text {Parkinson }}$ oder $\mathrm{AHB}_{\text {Alzheimer }}(\mathrm{F}=2,925 ; p<0,10)$. Signifikante Unterschiede zeigen sich bei den der Pflegeleistung hinterlegten Pflegestufen $\left(\chi^{2}=7,502 ; p<0,05\right)$ : $\mathrm{AHB}_{\text {Parkinson }}$ weisen im Mittel höhere Pflegestufen (Median: Stufe II) auf als $\mathrm{AHB}_{\text {Alzheimer }}$ (Median: Stufe I-II) und $\mathrm{AHB}_{\text {Tumor }}$ (Median: Stufe I). Während die Anzahl zusätzlicher Diagnosen zwischen Personen mit Parkinson und Tumorerkrankungen kaum differiert, werden bei jenen mit Alzheimer-Demenz deutlich weniger Komorbiditäten diagnostiziert $\quad(\mathrm{F}=13,859 ; \quad p<0,001)$. Hinsichtlich des Herkunftsorts vor dem Einzug in das Altenheim unterscheidet sich die Bewohnergruppe mit Morbus Parkinson stark von den beiden anderen. Menschen mit Parkinson werden deutlich seltener von der eigenen Häuslichkeit in das Altenheim transferiert $\left(\chi^{2}=12,361 ; p<0,05\right)$. Zudem variiert
In dieser Studie wurden drei Bewohnergruppen verglichen, deren primäre $\mathrm{Ge}$ meinsamkeit das Leben im Altenheim ist. Mit einem Fokus auf Schmerz bzw. Schmerztherapie wurden neben den bei- den neurodegenerativen Erkrankungen Morbus Alzheimer und Morbus Parkinson auch Tumorerkrankungen in die Sekundärdatenanalyse eingeschlossen. Die Bewohnergruppe mit onkologischen Erkrankungen wurde dabei als implizite „Referenzgruppe“ definiert, bei welcher das Phänomen Schmerz hinlänglich bekannt ist und diesbezüglich anerkannte und verbreitete Schmerzmanagementstrategien bestehen.

Ein Hauptergebnis der Untersuchung besteht darin, dass in allen untersuchten Bewohnergruppen eine hohe Prävalenz von Schmerzen festgestellt wurde. Schmerzen zeigten sich zudem unabhängig von der kognitiven Beeinträchtigung, die bei allen Gruppen zwar unterschiedlich, aber insgesamt recht deutlich ausgeprägt war. Dies lässt sich dahingehend interpretieren, dass die Schmerzevaluation in gewisser Weise losgelöst von den kognitiven Fähigkeiten gesehen und ein potenzielles Risiko von unerkannten Schmerzen in allen Gruppen generell angenommen werden sollte. Mitunter verdeutlichen diese Ergebnisse die vielfach postulierte Notwendigkeit einer initialen, regelhaften und differenzierten Schmerzeinschätzung unter Rückgriff auf zielgruppenspezifische Assessmentinstrumente [27]. So werden in einer kürzlich veröffentlichten Arbeit die Personenzentriertheit, das Bewusstsein sowie Schulungen über Schmerzthemen, die Regelhaftigkeit pflegerischer Maßnahmen, das Vorhandensein klarer Verantwortungsstrukturen und das Kernelement Kommunikation als die wichtigsten Aspekte des Schmerzmanagements bei Menschen mit Demenz angeführt [28]. Diese Forderungen können im Altenheimkontext im Grunde ebenso für Menschen mit Morbus Parkinson und Tumorerkrankungen unabhängig von kognitiven Veränderungen gelten.

Weitere signifikante Ergebnisse waren, dass bei Menschen mit Morbus Parkinson im Vergleich zu den anderen Bewohnergruppen die geringsten Schmerzen fremdeingeschätzt wurden, dass ebendiese Bewohner aber gleichzeitig die höchste Pflegestufe und den längsten Aufenthalt in Pflegeheimen hatten und in diesem Sinn die Gruppe mit dem größten Pflegeaufwand darstellten. 
Schmerzen haben beim Morbus Parkinson vielfältige Ursachen und sind oft direkt mit dem Progress der Grunderkrankung assoziiert. In der Regel sollte zunächst versucht werden, durch Anpassung der Parkinson-Therapie die Symptome wie Minderbewegung und Rigor zu verbessern, da diese häufige Schmerzursachen darstellen. Erst in zweiter Instanz sollen Schmerzmedikamente zum Einsatz kommen. Hierbei ist besonders darauf $\mathrm{zu}$ achten, dass keine Halluzinationen ausgelöst oder bestehende Halluzinationen verstärkt werden. Vor allem Belastungsschmerzen gelten in dieser Gruppe als weit verbreitet - nicht zuletzt sind diese auch häufig durch eine assoziierte Minderbeweglichkeit mitbedingt [29]. Für Menschen mit Morbus Parkinson stellt offensichtlich ein Aufenthalt im Krankenhaus oder in einer Kurzzeitpflege (in dieser Studie unter "Andere“ subsumiert) das "Tor“ zum Heimaufenthalt dar. Parkinson-Patienten werden bei Verschlechterung der Bewegungsstörung oder bei Auftreten von Sekundärphänomenen wie Pneumonie oder Delir stationär aufgenommen [30]. Dass der stationären Aufnahme dann eine Verlegung in ein Altenheim folgt, zeigt einerseits die oft fehlende Besserung der Symptome, andererseits kann das auch auf Überlastung von pflegenden Angehörigen, die sich dann letztendlich doch für eine Pflegeheimaufnahme entscheiden, zurückgeführt werden. Ein wichtiges Ergebnis ist zudem, dass bei der Gruppe der Bewohner mit Morbus Alzheimer die meisten auf das Vorkommen von Schmerzen hindeutenden Verhaltensmerkmale beobachtet wurden, diese Gruppe am häufigsten von zu Hause oder aus dem Krankenhaus in das Pflegeheim kam und insgesamt gesehen die wenigsten Zusatzdiagnosen aufwies. Die geringe Anzahl von $\mathrm{Zu}$ satzdiagnosen ist überraschend, da es sich um alte Menschen mit den üblichen kardiovaskulären Vorerkrankungen handelt. Hier stellt sich die Frage, ob die Zusatzdiagnosen beim Eintritt in das Altenheim so in den Hintergrund getreten sind, dass sie nicht mehr aufgeführt werden und damit auch keiner Kontrolle mehr unterliegen. Die Diagnose der Schmerzsymptome ist aber durch- aus gewährleistet, denn diese machen sich im Pflegealltag auch bemerkbar. Dass Bewohner mit Morbus Alzheimer höhere Schmerzen zu haben scheinen als Bewohner mit onkologischen Erkrankungen ist eventuell auch darauf zurückzuführen, dass bei onkologischen Erkrankungen qua Routine gleich bei Aufnahme in das Altenheim eine intensive Schmerzevaluation vorgenommen wird. Durch dieses Initialassessment werden Schmerzen dann eher antizipiert und ein regelmäßiges Anpassen der notwendigen Medikamente kann erfolgen.

\section{Limitationen}

$\mathrm{Zu}$ den wesentlichen Limitationen der Studie, die in unterschiedlichen Pflegeheimen durchgeführt wurde, zählt die Begrenzung der Untersuchung auf die Stadt Münster, Nordrhein-Westfalen. Zudem handelt es sich um kein randomisiertes Selektionsprozedere. Obwohl die wesentlichen Populationsparameter in der Stichprobe ähnlich ausgeprägt sind, limitiert das die Verallgemeinerung der Ergebnisse. Weiter befinden sich unter den untersuchten Personen überwiegend Frauen, was zwar der Bewohnerstruktur in Altenheimen nahekommt, aber vor dem Hintergrund kleinerer Gruppenfallzahlen dazu führt, dass ein gewisser Selektionbias nicht ausgeschlossen werden kann.

\section{Schlussfolgerung}

Die Untersuchung macht auf eine beachtenswert hohe Schmerzprävalenz aller untersuchten Altenheimbewohner aufmerksam. Als übergeordnete Konsequenz ist daher dringend zu fordern, für eine ausreichende Personalabdeckung durch gut geschulte und damit qualifizierte Pflegende zu sorgen. Die Pflege von Menschen mit neurologischen Erkrankungen und insbesondere in der letzten Lebensphase ist an sich schon herausfordernd genug. Nicht erkannte oder nicht adäquat behandelte Schmerzen sollten nicht dazu führen, dass der Heimaufenthalt und der letzte Lebensabschnitt von Betroffenen sowie in weiterer Konsequenz die professionelle Pflegetätigkeit zusätzlich weiter belastet und komplexer werden. Sehr wesentlich erscheint dabei eine gute Teamarbeit zwischen den Pflegenden vor Ort und den behandelnden Fach- (etwa Neuro- und Onkologen) sowie Hausärzten. Im Hinblick auf die teils deutlichen kognitiven Beeinträchtigungen der untersuchten Altenheimbewohner bzw. der Altenheimpopulation im Allgemeinen ist es auch essenziell, unter allen Beteiligten abgestimmte Prozesse und Strukturen zu schaffen, in denen Schmerzsituationen durch Betroffene so kommuniziert werden können, dass sie adäquat einschätzbar und somit behandelbar werden.

\section{Fazit für die Praxis}

\section{- Bei allen Bewohnergruppen zei- gen sich Hinweise auf vorhandene Schmerzproblematiken und hohe Schmerzprävalenzen. \\ - Die Versorgungssituation von Al- tenheimbewohnern mit Morbus Parkinson, Morbus Alzheimer und onkologischen Erkrankungen unter- scheidet sich teils deutlich. \\ - Interprofessionelle und spezifisch an die Bedürfnisse von Bewohnern mit Parkinson und Alzheimer angepasste Schmerz- und Palliativversorgungs- prozesse sind gefordert.}

\section{Korrespondenzadresse

Mag. P. Kutschar
Institut für Pflegewissenschaft
und -praxis, Paracelsus
Medizinische Privatuniversität
Strubergasse 21,
5020 Salzburg, Österreich
patrick.kutschar@pmu.ac.at

Funding. Open access funding provided by Paracelsus Medical University.

\section{Einhaltung ethischer Richtlinien}

Interessenkonflikt. Das Hauptprojekt "Aktionsbündnis Schmerzfreie Stadt Münster" wurde von der Fa. Mundipharma GmbH finanziell unterstützt. In ihrer Funktion als Förderer nahm die Mundipharma GmbH keinerlei Einfluss auf die wissenschaftliche Erhebung, Auswertung der Projektdaten oder Publikation von Ergebnissen. P. Kutschar, K. Lex, J. Osterbrink und S. Lorenzl geben an, dass kein Interessenkonflikt besteht. 
Dieser Beitrag beinhaltet keine von den Autoren durchgeführten Studien an Menschen oder Tieren.

Open Access. Dieser Artikel wird unter der Creative Commons Namensnennung 4.0 International Lizenz (http://creativecommons.org/licenses/by/4.0/deed. de) veröffentlicht, welche die Nutzung, Vervielfältigung, Bearbeitung, Verbreitung und Wiedergabe in jeglichem Medium und Format erlaubt, sofern Sie den/die ursprünglichen Autor(en) und die Quelle ordnungsgemäßnennen, einen Linkzur Creative Commons Lizenz beifügen und angeben, ob Änderungen vorgenommen wurden

\section{Literatur}

1. Statistisches Bundesamt (2017) Pflegestatistik 2015. Pflege im Rahmen der Pflegeversicherung. Deutschlandergebnisse

2. Becker-Ebel J, Behrens C, Davids G et al (2012) Palliative Care in Pflegeheimen. Wissen und Handeln für Altenpflegekräfte. Schlütersche Verlagsgesellschaft, Hannover

3. Kostrzewa S (2010) Palliative Pflege von Menschen mit Demenz. Huber, Bern

4. Osterbrink J, Hufnagel M, Kutschar P et al (2012) Die Schmerzsituation von Bewohnern in der stationären Altenhilfe. Schmerz 26:27-35

5. Knipping C (2007) Lehrbuch Palliative Care. Huber, Bern (zitiertaus S. Pleschberger, 2005)

6. Rodin MB (2008) Cancer patients admitted to nursing homes: what do we know? J Am Med Dir Assoc 9(3):149-156

7. Robert Koch Institut (2015) Krebs in Deutschland 2011/2012. Beiträge zur Gesundheitsberichterstattung des Bundes Berlin. http://www.gekid.de/ Doc/krebs_in_deutschland_2015.pdf.Zugegriffen:7.Juni 2017

8. Deutsche Krebsgesellschaft, Deutsche Krebshilfe (2015) Arbeitsgemeinschaft der Wissenschaftlichen Medizinischen Fachgesellschaften e.V., Leitlinienprogramm Onkologie

9. Davies E, Higginson IJ (2004) Better palliative care for older people. http://www.euro.who. int/_data/assets/pdf_file/0009/98235/E82933. pdf.Zugegriffen: 18. Juni 2017

10. Bukki J, Unterpaul T, Nubling G et al (2014) Decision making at the end of life - cancer patients' and their caregivers' views on artificial nutrition and hydration. Support Care Cancer 22(12):3287-3299

11. Schäufele $M$, Köhler $L$, Hendlmeier I et al (2013) Prävalenz von Demenzen und ärztliche Versorgung in deutschen Pflegeheimen: Eine bundesweite repräsentative Studie. Psychiatr Prax 40:200-206

12. Deutsches Zentrum für Neurodegenerative Erkrankungen (DZNE) (2017) Neurodegeneration in einer alternden Gesellschaft. https://www.dzne. de/wissenschaft-gesellschaft.html. Zugegriffen: 8. Juni 2017

13. Kojer M (2006) Demenz und Palliative Care. In: Kränzle S, Schmid S, Seeger C (Hrsg) Palliative Care. Handbuch für Pflege und Begleitung. Springer, Berlin, Heidelberg, S249-259

14. Kojer M, Schmidl M (2011) Demenz und Palliative Geriatrie in der Praxis. Heilsame Betreuung unheilbar demenzkranker Menschen. Springer, New York

15. Bartholomeyczik S, Halek M (2017) Pflege von Menschen mit Demenz. In: Jacobs K et al (Hrsg) Pflegereport 2017. Schwerpunkt: Die Versorgung derPflegebedürftigen. Schattauer, Stuttgart
16. Oliver DJ, Borasio GD, Caraceni A et al (2016) A consensus review on the development of palliative care for patients with chronic and progressive neurological disease. Eur JNeurol 23(1):30-38

17. Bower JH, Maraganore DM, McDonnell SK et al (1999) Incidence and distribution of parkinsonism in Olmsted County, Minnesota, 1976-1990. Neurology 52(6):1214-1220

18. Higginson IJ, Gao W, Saleem TZ et al (2012) Symptoms and quality of life in late stage Parkinson syndromes: a longitudinal community study of predictive factors. PLoS ONE 7(11):e46327

19. European Association Palliative Care (EAPC) (1989) Definition palliative care. http://www.eapcnet. eu/Corporate/AbouttheEAPC/DefinitionandAims. aspx. Zugegriffen: 9. Juni 2017

20. Temel JS, Greer JA, Muzikansky A et al (2010) Early palliative care for patients with metastatic non-small-cell lung cancer. $\mathrm{N}$ Engl J Med 363(8):733-742

21. Safarpour D, Thibault DP, DeSanto CL et al (2015) Nursing home and end-of-life care in Parkinson disease. Neurology 85(5):413-419

22. Mitchell SL, Kiely DK, Hamel MB (2004) Dying with advanced dementia in the nursing home. Arch Intern Med 164(3):321-326

23. Ivemeyer D, Zerfaß R (2006) Demenztests in der Praxis. Ein Wegweiser, 2. Aufl. Urban \& Fischer, München, Jena

24. Chibnall JT, Tait RC (2001) Pain assessment in cognitively impaired and unimpaired older adults: a comparison of four scales. Pain 92(1):173-186

25. Schuler MS, Becker S, Kaspar R et al (2007) Psychometric properties of the German "Pain Assessment in Advanced Dementia Scale" (PAINAD$\mathrm{G}$ ) in nursing home residents. J Am Med Dir Assoc 8(6):388-395

26. Zwakhalen S, van der Steen J, Najim MD (2011) Which score represents pain on the observational PAINAD pain scale for dementia patients? Int Psychogeriatr 23:S48-S49

27. Deutsches Netzwerk für Qualitätsentwicklung in der Pflege (DNQP) (2015) Expertenstandard Schmerzmanagement in der Pflege bei chronischen Schmerzen. DNQP, Osnabrück

28. Corbett A, Nunez KM, Smeaton E et al (2016) The landscape of pain management in people with dementia living in care homes: a mixed methods study. Int J Geriatr Psychiatry 31 (12):1354-1370

29. Thompson T, Gallop K, Correll CU et al (2017) Pain perception in Parkinson's disease: a systematic review and meta-analysis of experimental studies. Ageing Res Rev 35:74-86

30. Weerkamp NJ, Tissingh G, Poels PJ et al (2014) Parkinson disease in long term care facilities: a review of the literature. J Am Med Dir Assoc 15(2):90-94
Hier steht eine Anzeige. Springer 